\title{
CMB dipole asymmetry from a fast roll phase
}

\author{
Anupam Mazumdar and Lingfei Wang \\ Physics Department, Lancaster University, Lancaster LA1 4YB, UK
}

\begin{abstract}
The observed CMB (cosmic microwave background) dipole asymmetry cannot be explained by a single field model of inflation - it inevitably requires more than one field where one of the fields is responsible for amplifying the super-Hubble fluctuations beyond the pivot scale. Furthermore the current constraints on $f_{\mathrm{NL}}$ and $\tau_{\mathrm{NL}}$ require that such an amplification cannot produce large non-Gaussianity. In this paper we propose a model to explain this dipole asymmetry from a spectator field, which is responsible for generating all the curvature perturbations, but has a temporary fast roll phase before the Hubble exit of the pivot scale. The current data prefers spectator scenario because it leaves no isocurvature perturbations. The spectator model will also satisfy the well-known constraints arising from quasars, and the quadrupole and octupole of the CMB.
\end{abstract}




\section{Contents}

1 Introduction 1

2 CMB dipole asymmetry 2

3 Slow roll evolution with two fields 3

3.1 Fluctuations of a light field 4

3.2 Lack of dipole asymmetry 5

4 Tachyonic fast roll phase of a spectator 5

4.1 Enhancing the perturbations 6

4.2 Generating CMB dipole asymmetry 8

5 Known constraints $\quad 10$

$\begin{array}{lll}5.1 \text { Quasars } & 10\end{array}$

$\begin{array}{ll}5.2 \text { Quadrupole and octupole } & 10\end{array}$

6 A viable model $\quad 11$

7 Conclusion $\quad 12$

\section{Introduction}

The primordial inflation matches all the known predictions for the cosmic microwave background (CMB) temperature anisotropy [1-3]. However recent observations from WMAP $[4,5]$ and Planck [6] found a dipole asymmetry in the CMB power spectrum with $3 \sigma$ significance. The origin of this asymmetry is hard to explain within a single field model of inflation. It was first pointed out in Refs. $[7,8]$ that an initial perturbation with a scale larger than the CMB pivot scale could be responsible for the asymmetry by favoring a certain direction. This would inevitably require more than one field dynamics during inflation, because a single field inflation can not give rise to a large non-Gaussianity required to explain the dipole asymmetry ${ }^{1}$.

It is also possible that there could be more fields during inflation, which are responsible for creating large non-Gaussianity with opposite signs, i.e. $\pm f_{\mathrm{NL}}[16]$. Their fine cancellations could yield not only the dipole asymmetry, but would also match the current limits on $f_{\mathrm{NL}}=2.7 \pm 11.6$ (at $2 \sigma$ ). Similarly there are other interesting suggestions, see [17-22]. Other attempts have also been made to address multiple CMB anomalies altogether in [23-25].

As it was pointed out in Ref. [16], one would require a violation of slow-roll dynamics for the fields responsible for creating super-long wavelength perturbations. One simple way of obtaining this would be via a brief period of fast roll phase [26], before the relevant

\footnotetext{
${ }^{1}$ One has to further make sure that there is no residual isocurvature perturbations [9], and the end of inflation solely excites the Standard Model relativistic degrees of freedom [10], since there is no evidence of dark radiation from Planck [3]. This already constrains models of inflation, which can explain both CMB perturbations and ensure right thermal history of the universe [11]. Only visible sector models of inflation, where inflation is embedded within a Standard Model gauge theory or its minimal supersymmetric extensions can ensure a consistent scenario for a successful inflationary paradigm, see Refs. [12-15] and [10].
} 
perturbations have left the Hubble patch. The fast roll phase enhances the perturbations on scales larger than the pivot scale, but leaves the pivot and smaller scales unaffected. However, one has to check whether the dipole asymmetry can contaminate the quadrupole and the octupole.

In this paper we will study all these constraints within a simple scenario where we have inflaton within a visible sector, and a spectator field which decays during inflation but after sourcing the large scale fluctuations, as discussed in Refs. [27, 28] ${ }^{2}$.

We give a brief overview on the CMB dipole asymmetry in section 2. We study the dipole asymmetry for a slow roll spectator field in section 3 . The enhancement in the perturbations leading to the dipole asymmetry has been computed in section 4 . We consider the known constraints in section 5 , and provide a viable example in section 6 . We conclude in section 7 .

\section{CMB dipole asymmetry}

The dipole asymmetry in the CMB power spectrum can be modeled by the directional dependence $\hat{\mathbf{n}}$ in the temperature fluctuations, given by $^{3}$ (see for instance [5])

$$
\delta T(\hat{\mathbf{n}})=(1+A \hat{\mathbf{p}} \cdot \hat{\mathbf{n}}) \overline{\delta T}(\hat{\mathbf{n}}),
$$

where the dipole asymmetry is along the direction $\hat{\mathbf{p}}$, and its strength is $0<A \ll 1$. After extracting the dipole asymmetry, $\overline{\delta T}(\hat{\mathbf{n}})$ then has an isotropic Gaussian distribution. If we pick a local patch in the direction $\hat{\mathbf{n}}$ on the CMB map, and calculate the CMB power spectrum only in this patch, it will also acquire a directional dependence (neglecting $\mathcal{O}\left(A^{2}\right)$ ),

$$
P_{\delta T}(\hat{\mathbf{n}})=(1+2 A \hat{\mathbf{p}} \cdot \hat{\mathbf{n}}) P_{\delta T} .
$$

This directional dependence becomes most significant when we compare the two opposite directions, $\hat{\mathbf{n}}=\hat{\mathbf{p}}$ and $\hat{\mathbf{n}}=-\hat{\mathbf{p}}$. Their relative difference is given by

$$
\frac{P_{\delta T}(\hat{\mathbf{p}})-P_{\delta T}(-\hat{\mathbf{p}})}{P_{\overline{\delta T}}}=4 A .
$$

The latest Planck observations [6] constrain the value, $A=0.07 \pm 0.02$, while confirming the previous analysis on the WMAP data $[4,5]$. Since the temperature anisotropy is seeded by the primordial curvature perturbations, it is straightforward to think that its dipole asymmetry may share the same origin.

The dipole asymmetry may arise from a scalar field, $\sigma$, which partly or totally contributes to the primordial curvature perturbations, and has a non-uniform background value at the Hubble exit. This can be caused by the initial field perturbations of $\sigma$, whose wavelengths are much larger than that of the pivot scale, as argued in Refs. [7, 8, 16, 18, 20, 21].

Therefore, we can assume such initial field perturbations of $\sigma$ can create the difference $\Delta \sigma$ in its background evolutions of the two local universe patches along the $\hat{\mathbf{p}}$ and $-\hat{\mathbf{p}}$

\footnotetext{
${ }^{2}$ In principle a curvaton scenario [29-32] could also provide the dipole asymmetry within our mechanism, but since the curvaton decays after inflation, it can potentially create large non-Gaussianity and large isocurvature fluctuations. Furthermore, one has to ensure that the curvaton decays into the standard model degrees of freedom $[10,33]$. In this respect a spectator field which decays during inflation could be much more helpful for model building [10, 27, 28], because it does not leave any isocurvature fluctuations, and naturally provides smaller non-Gaussianity than the curvaton, while the inflaton could be within a visible sector.

${ }^{3}$ We use bold symbols to indicate vectors.
} 
directions. This will result in the difference in the power spectrum of the primordial curvature perturbations, $\zeta$, by an amount

$$
\Delta P_{\zeta}=\frac{\partial P_{\zeta}}{\partial \sigma} \Delta \sigma
$$

Since $P_{\delta T} \propto P_{\zeta}$ at the linear order, from eq. (2.3) we know

$$
A=\frac{\Delta P_{\zeta}}{4 P_{\zeta}}=\frac{1}{4} \frac{\partial P_{\zeta}}{P_{\zeta} \partial \sigma} \Delta \sigma
$$

We can write $A$ as a function of the power spectrum of the curvature perturbations $P_{\zeta}$ and that of the field perturbations $P_{\delta \sigma_{*}}$, as well as the primordial local bispectrum $f_{\mathrm{NL}}$ or trispectrum $\tau_{\mathrm{NL}}$, see $[16,18]$. There are two ways of satisfying small $f_{\mathrm{NL}}$ and the observed dipole asymmetry.

- Inflation and the spectator: If $\sigma$ is a spectator field, which is the only source of the primordial curvature perturbations, the amount of CMB dipole asymmetry can be shown as $[16,18]$ :

$$
A=\frac{3}{5} \frac{|\Delta \sigma|}{\sqrt{P_{\delta \sigma_{*}}}}\left|f_{\mathrm{NL}}\right| \sqrt{P_{\zeta}} .
$$

The latest Planck observations $[3,6,34]$ give the central values $P_{\zeta}=2.196 \times 10^{-9}$, $A=0.07$. The local bispectrum is constrained by $f_{\mathrm{NL}}=2.7 \pm 5.8$, which can provide an upper bound as $\left|f_{\mathrm{NL}}\right|<14.3$ (@ $>95 \%$ C.L.). To achieve $A=0.07$, we would require:

$$
\frac{|\Delta \sigma|}{\sqrt{P_{\delta \sigma_{*}}}}>174
$$

- Inflaton, spectator, and the other: When the spectator $\sigma$ coexists with other sources of curvature perturbations, then they may generate opposite local bispectra $\pm f_{\mathrm{NL}}$ ( individual contributions could be large ), which mostly cancel to yield a small total $f_{\mathrm{NL}}$. The negative values of $f_{\mathrm{NL}}$ may come from preheating [35-37]. This can enhance the CMB dipole asymmetry, to the maximum extent as [16]

$$
A<\frac{|\Delta \sigma|}{2 \sqrt{P_{\delta \sigma_{*}}}} \sqrt{\tau_{\mathrm{NL}} P_{\zeta}}
$$

From the 95\% CL upper bound on $\tau_{\mathrm{NL}}<2800$, we only need a weaker field difference, $\Delta \sigma$, in order to generate the CMB dipole asymmetry $A=0.07$, as

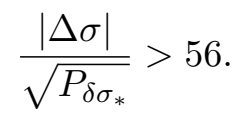

In this paper we will be focusing on how to excite the $\sigma$ field such that one can obtain large values of overall $\Delta \sigma$.

\section{Slow roll evolution with two fields}

It has been briefly discussed in [16] that if the slow roll approximations are well satisfied for a curvaton or spectator field $\sigma$, its initial perturbations of any single wavelength would not give rise to any significant $\mathrm{CMB}$ asymmetry. 
In the simplest scenario, we can think of a specator field $\sigma$ as the source of curvature perturbations. The inflation is dominated by another field $\phi$, which rolls very slowly, providing a nearly constant Hubble rate of expansion $H$ during inflation. For minimalism, we assume $\sigma$ has negligible interactions with the inflaton, so any perturbation in $\sigma$ will not affect the inflaton, and will only convert to curvature perturbations well after the corresponding Hubble exit. Its energy density should also be subdominant than that of the inflaton's. The action is given by:

$$
S=\int \mathrm{d}^{4} x \sqrt{\left|-g_{\mu \nu}\right|}\left(-\frac{1}{2} \partial_{\mu} \sigma \partial^{\mu} \sigma-\frac{1}{2} m^{2} \sigma^{2}+\mathcal{L}_{\phi}\right),
$$

where $g_{\mu \nu}=\operatorname{diag}\left(-1, a^{2}, a^{2}, a^{2}\right)$ with $a(t)$ being the scale factor, and $\mathcal{L}_{\phi}$ is the Lagrangian density for the inflaton, which may arise from the visible sector. We assume $m \ll H$ during inflation.

\subsection{Fluctuations of a light field}

We can separate the inhomogeneous part of $\sigma$ as its perturbations $\delta \sigma(x, t)$, by writing $\sigma(x, t)=\sigma(t)+\delta \sigma(x, t)$. From the total action eq. (3.1), the perturbation $\delta \sigma(x, t)$ yields to its equation of motion (assuming $m^{2} \ll H^{2}$ )

$$
\ddot{\delta \sigma}+3 H \dot{\delta \sigma}-\partial_{i} \partial^{i} \delta \sigma=0,
$$

where dot means taking derivative w.r.t time $t$. A Fourier transformation into the momentum space $\delta \sigma_{\mathbf{k}}(t)$ then gives

$$
\ddot{\delta} \sigma_{\mathbf{k}}+3 H \dot{\delta \sigma_{\mathbf{k}}}+\frac{k^{2}}{a^{2}} \delta \sigma_{\mathbf{k}}=0 .
$$

For the sub-Hubble modes with $k^{2} \gg a^{2} H^{2}$, we define the conformal time $\mathrm{d} \tau \equiv \mathrm{d} t / a$, and $u_{\mathbf{k}} \equiv a \delta \sigma_{\mathbf{k}}$. We use prime to indicate $\mathrm{d} / \mathrm{d} \tau$, and the equation of motion eq. (3.3) can be rewritten as

$$
u_{\mathbf{k}}^{\prime \prime}+\left(k^{2}-2 a^{2} H^{2}\right) u_{\mathbf{k}}=0, \quad \text { for } k^{2} \gg a^{2} H^{2} .
$$

For the super-Hubble modes with $k^{2} \ll a^{2} H^{2}$, we similarly define $\psi_{\mathbf{k}} \equiv a^{\frac{3}{2}} \sigma_{\mathbf{k}}$. This reduces eq. (3.3) to

$$
\ddot{\psi}_{\mathbf{k}}-\left(\frac{9}{4} H^{2}-\frac{k^{2}}{a^{2}}\right) \psi_{\mathbf{k}}=0, \quad \text { for } k^{2} \ll a^{2} H^{2} .
$$

We do not specify the inflation model, but instead just assume $H$ remains constant throughout inflation. Then the universe will expand exponentially, but this will only make slow changes in the corresponding effective masses of eq. (3.4) and eq. (3.5). The above solutions can be recast into

$$
\left\langle\left|\delta \sigma_{\mathbf{k}}(N)\right|^{2}\right\rangle=e^{-2 \int_{N_{0}}^{N} \alpha_{\mathbf{k}}(N) \mathrm{d} N}\left\langle\left|\delta \sigma_{\mathbf{k}}\left(N_{0}\right)\right|^{2}\right\rangle
$$

where $N \equiv \ln a$ is the number of e-folds of universe expansion, which we use as the proper time. After defining $N_{k}$ as

$$
k^{2}=2 e^{2 N_{k}} H^{2},
$$

we can write $\alpha_{\mathbf{k}}(N)$ as

$$
\left.\alpha_{\mathbf{k}}(N)\right|_{s r}= \begin{cases}1, & \text { for } N_{k} \geq N,\left(k^{2} \geq 2 a^{2} H^{2}\right) \\ \frac{3}{2}-\sqrt{\frac{9}{4}-2 e^{2\left(N_{k}-N\right)}}, & \text { for } N_{k}<N,\left(k^{2}<2 a^{2} H^{2}\right),\end{cases}
$$

where we use the subscript "sr" to indicate the slow roll case. 


\subsection{Lack of dipole asymmetry}

From eq. (3.8), we see that as the universe expands, any mode $\mathbf{k}$ will reach $N \gg N_{k}$, where $\left.\alpha_{\mathbf{k}}\right|_{s r} \rightarrow 0$. This means the perturbations $\delta \sigma_{\mathbf{k}}$ will gradually freeze. Based on the solution eq. (3.8), the canonical quantization then gives the simple relation $\left.\left\langle\left|\delta \sigma_{\mathbf{k}}(N)\right|^{2}\right\rangle\right|_{s r} \propto k^{-3}$ for $N \gg N_{k}$. This corresponds to the perturbation in the $x$ space

$$
\left\langle|\delta \sigma(x)|^{2}\right\rangle=\int\left\langle\left|\delta \sigma_{\mathbf{k}}\right|^{2}\right\rangle \mathrm{d}^{3} \mathbf{k}=\int P_{\delta \sigma_{k}} \mathrm{~d} \ln k,
$$

and here we have a scale invariant power spectrum $\left.P_{\delta \sigma_{\mathbf{k}}}\right|_{s r} \propto k^{0}$.

The perturbations will also generate a gradient along any arbitrary $z$ direction

$$
\left\langle\left|\frac{\partial}{\partial z} \delta \sigma(x)\right|^{2}\right\rangle=\int\left\langle\left|\delta \sigma_{\mathbf{k}}\right|^{2}\right\rangle k_{z}^{2} \mathrm{~d}^{3} \mathbf{k}=\int P_{\delta \sigma_{k}} \frac{k^{2}}{2} \mathrm{~d} \ln k .
$$

This gradient will source the field difference between the opposite sides on the last scattering surface along the $z$ direction, by an amount

$$
\Delta \sigma^{2}=4 r_{\mathrm{ls}}^{2}\left\langle\left|\frac{\partial}{\partial z} \delta \sigma(x)\right|^{2}\right\rangle,
$$

where $r_{\mathrm{ls}}$, our distance to the last scattering surface, is defined by

$$
r_{\mathrm{ls}}=\frac{1}{a_{*} H_{*}}=\frac{e^{-N_{*}}}{H} .
$$

Therefore, the relative strength of the field difference becomes

$$
\left.\frac{\Delta \sigma^{2}}{P_{\delta \sigma_{*}}}\right|_{s r}=\int 2 r_{\mathrm{ls}}^{2} k^{2} \mathrm{~d} \ln k=\int 4 e^{2\left(N_{k}-N_{*}\right)} \mathrm{d} N_{k} .
$$

Note that only the scales much larger than the pivot scale can significantly contribute to the field difference $\Delta \sigma$ from their gradient contribution. This means the upper bound of the integral in eq. (3.13) should be $N_{k}<N_{*}$. For this reason, the exponential suppression inside the integral will give rise to

$$
\left.\frac{\Delta \sigma^{2}}{P_{\delta \sigma_{*}}}\right|_{s r}=2 e^{2\left(N_{k}-N_{*}\right)}<2 .
$$

This is insufficient to generate the observed CMB asymmetry, see eq. (2.9). We conclude that a light canonical slow roll $\sigma$ field cannot generate sufficient CMB asymmetry.

\section{Tachyonic fast roll phase of a spectator}

As we suggested earlier in Ref. [16], a violation in the slow roll conditions may produce sufficient CMB dipole asymmetry. It is well known that the perturbations can get enhanced during a tachyonic fast roll phase [26]. Therefore we wish to investigate how a tachyonic fast roll phase may enhance the CMB asymmetry. 


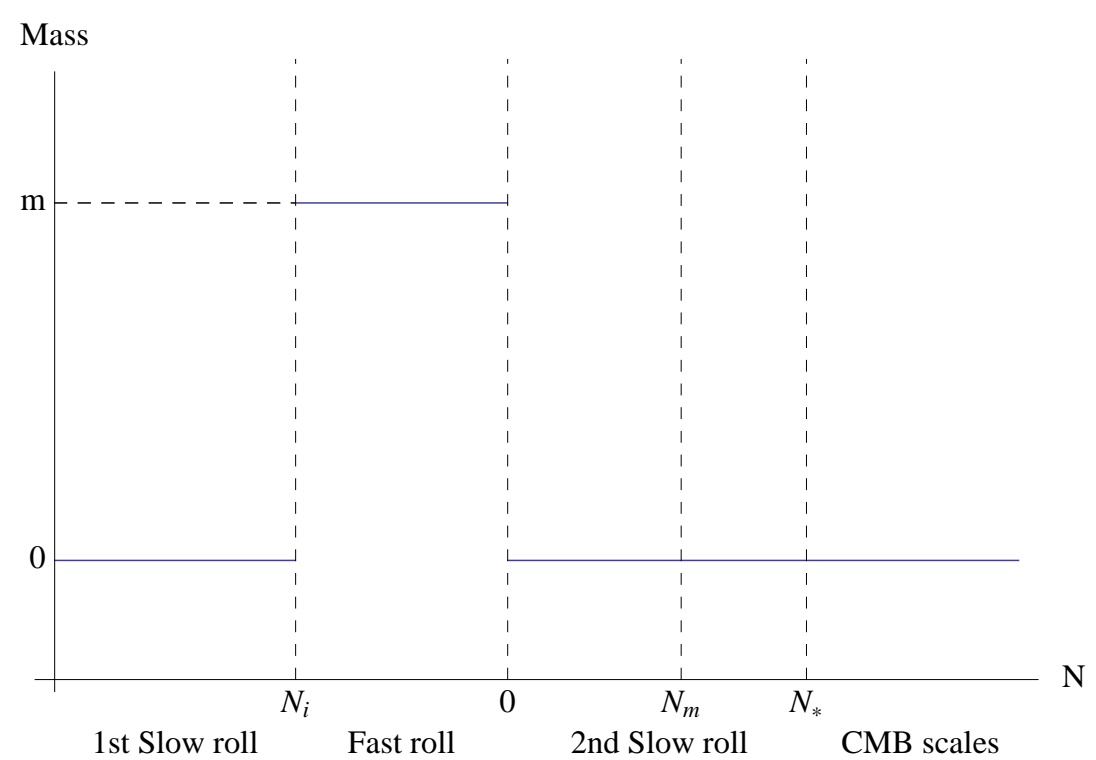

Figure 1. The hierarchy of scales, and the timeline of the tachyonic fast roll scenario. The tachyonic fast roll phase lasts during $N_{i}<N<0$.

Let us assume that the spectator field acquires a tachyonic mass for a brief period. The total action for the tachyonic phase can be given by

$$
S=\int \mathrm{d}^{4} x \sqrt{\left|-g_{\mu \nu}\right|}\left(-\frac{1}{2} \partial_{\mu} \sigma \partial^{\mu} \sigma+\frac{1}{2} m^{2} \sigma^{2}+\mathcal{L}_{\phi}\right),
$$

where we can also define $N_{m}$ to write $m$ w.r.t $H$ as

$$
m^{2}=\left(e^{2 N_{m}}-1\right) 2 H^{2} .
$$

Then we will get a typical timeline as in figure 1 . We choose $a=1$ i.e. $N=0$ for the end of the fast roll phase, and assume its beginning lies at $N_{i}<0$. The mass scale is indicated as $N_{m}>0$ in figure 1 . To prevent the fast roll phase from affecting the CMB spectrum, we need the pivot scale to satisfy $N_{*} \geq N_{m}$, as we will show shortly.

\subsection{Enhancing the perturbations}

We focus on the fast roll phase and follow the steps of section 3. The equation of motion for the perturbation $\delta \sigma$ in momentum space during the fast roll phase then becomes

$$
\ddot{\delta} \sigma_{\mathbf{k}}+3 H \dot{\delta} \sigma_{\mathbf{k}}+\left(\frac{k^{2}}{a^{2}}-m^{2}\right) \delta \sigma_{\mathbf{k}}=0
$$

Similar calculations will yield ${ }^{4}$

$$
\alpha_{\mathbf{k}}(N)= \begin{cases}1, & \text { for } N_{k} \geq N+N_{m}, \\ \frac{3}{2}-\sqrt{\frac{1}{4}+2\left(e^{2 N_{m}}-e^{2\left(N_{k}-N\right)}\right),} & \text { for } N_{k}<N+N_{m} .\end{cases}
$$

\footnotetext{
${ }^{4}$ Here we only inspect the simplest setup, i.e. the tachyonic mass $m$ is switched on and off instantly, and remains constant during the period. In more realistic scenarios, the mass $m$ may depend on time, by writing $m(N)$ or $N_{m}(N)$. The subsequent calculations can still be performed as long as the WKB approximation is applicable. Although the specific results will depend on $N_{m}(N)$, the general consequence will not change, i.e. the field perturbations $\delta \sigma_{\mathbf{k}}$ will be enhanced in a scale-dependent way.
} 


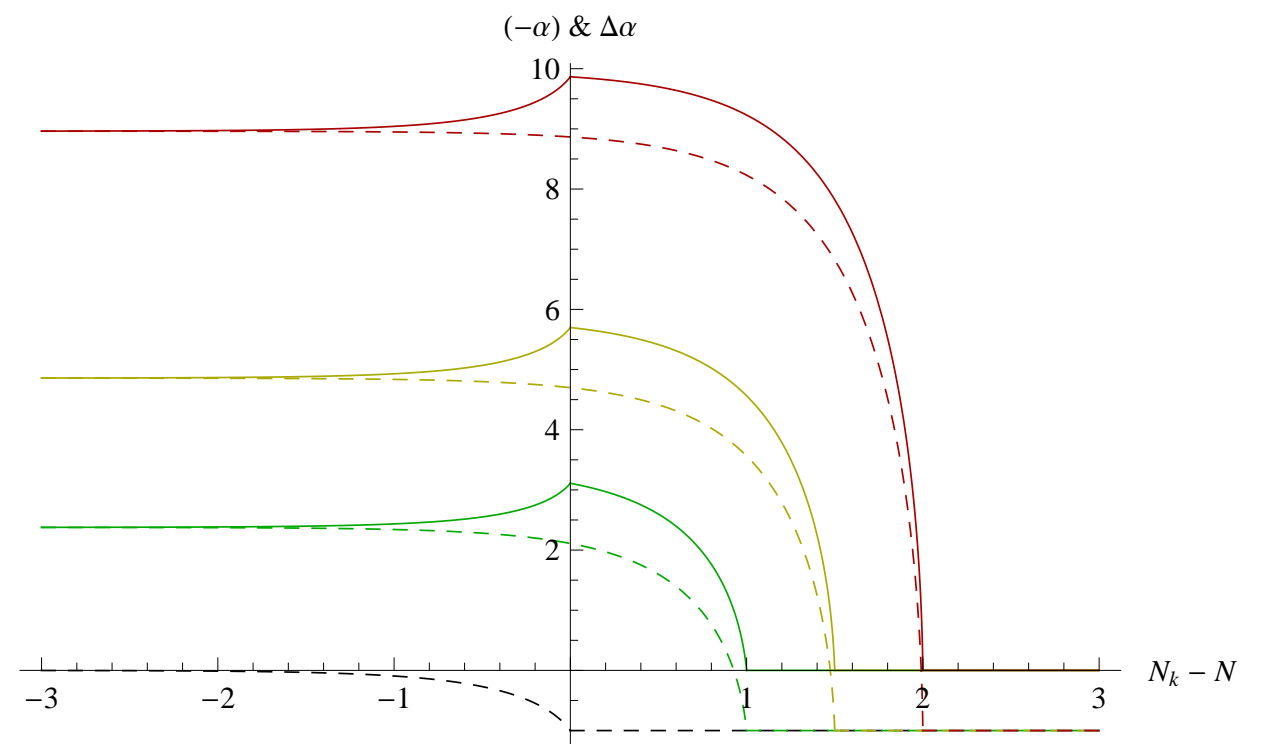

Figure 2. A demonstration of $-\alpha_{\mathbf{k}}(N)$ and the enhance rate $\Delta \alpha_{\mathbf{k}}(N)$ for some typical values of $N_{m}$. From bottom to top, black, green, yellow and red correspond to $N_{m}=0,1,1.5,2$ respectively. Dashed curves represent $-\alpha_{\mathbf{k}}(N)$, and solid ones represent $\Delta \alpha_{\mathbf{k}}(N)$.

By comparing the values of $\alpha_{\mathbf{k}}(N)$ for the two scenarios, eq. (3.8) and eq. (4.4), we see that the outcome is different. Although the sub-Hubble modes are always redshifted with the rate $\alpha_{\mathbf{k}}=1$ in both the cases, the sub/super-Hubble boundary is shifted to $N_{k}=N_{m}+N$ in the fast roll scenario. Therefore the modes within $N<N_{k}<N_{m}+N$, which would be regarded as sub-Hubble and redshifted as $\alpha_{\mathbf{k}}=1$ in the slow roll scenario, are now in the fast roll scenario super-Hubble, with a lower damping rate $\alpha_{\mathbf{k}}<1$. In this sense, when the tachyonic mass is present, the small scale modes $\left(N_{k}>N+N_{m}\right)$ are unaffected (with the same damping rate $\left.\alpha_{\mathbf{k}}=1\right)$, while the intermediate scales $\left(N<N_{k}<N+N_{m}\right)$ are enhanced compared to the slow roll case.

The large scale modes $\left(N_{k}<N\right)$ are also enhanced by the tachyonic mass comparatively, which is easy to see. Moreover, in the slow roll scenario we would always get $0<\alpha_{\mathbf{k}}<1$, but in the tachyonic fast roll scenario, it is possible to achieve $\alpha_{\mathbf{k}}<0$ when $N_{m}$ is sufficiently large, i.e. $m \gg H$. This corresponds to the case when the amplification by the tachyonic potential overcomes the expansion of the universe and the spatial inhomogeneities.

We can then write the enhance rate for the field perturbation $\delta \sigma_{\mathbf{k}}$, as the difference between the two damping rates,

$$
\left.\Delta \alpha_{\mathbf{k}}(N) \equiv \alpha_{\mathbf{k}}(N)\right|_{s r}-\alpha_{\mathbf{k}}(N) .
$$

Therefore we get the enhance rate for small, intermediate and large scales as

$$
\Delta \alpha_{\mathbf{k}}(N)= \begin{cases}0, & \text { for } N_{k} \geq N+N_{m}, \\ \sqrt{\frac{1}{4}+2\left(e^{2 N_{m}}-e^{2\left(N_{k}-N\right)}\right)}-\frac{1}{2}, & \text { for } N+N_{m}>N_{k} \geq N, \\ \sqrt{\frac{1}{4}+2\left(e^{2 N_{m}}-e^{2\left(N_{k}-N\right)}\right)}-\sqrt{\frac{9}{4}-2 e^{2\left(N_{k}-N\right)}}, & \text { for } N_{k}<N .\end{cases}
$$

This is plotted with some typical values of $N_{m}$ in figure 2 , from which we can see the enhancement can be quite significant $\Delta \alpha_{\mathbf{k}} \sim e^{N_{m}}$. Also, from eq. (4.6) we can see that the 


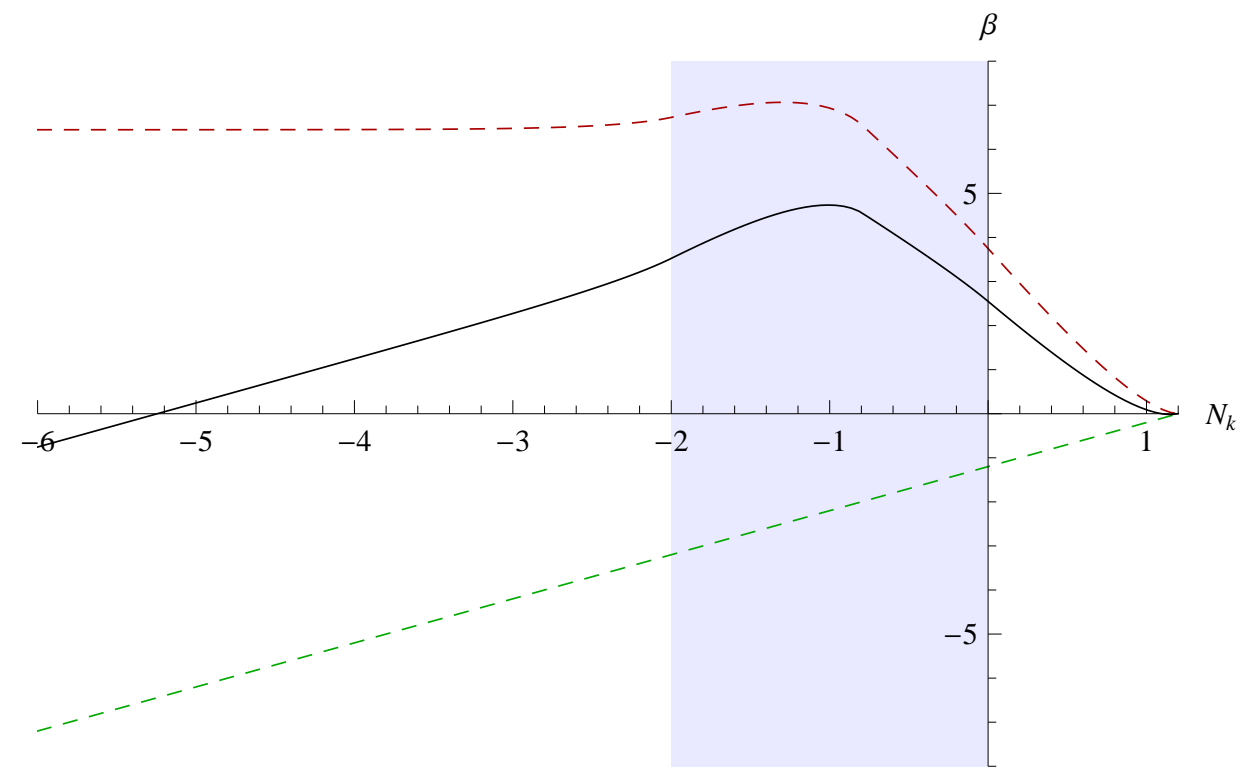

Figure 3. The enhancement $\beta$ is shown in the black solid curve, where its components $N_{k}-N_{m}$ and the integral in eq. (4.9) are shown in green and red dashed curves respectively. The blue shaded region is the number of e-folds during the fast roll phase. We have taken the parameter values $N_{i}=-2, N_{m}=1.2, N_{*}=2.2$.

small scales with $N_{k} \geq N+N_{m}$ are not affected by the fast roll phase. Remembering the fast roll phase lasts from $N=N_{i}<0$ to $N=0$, the scales $N_{k} \geq N_{m}$ will be totally unaffected, which is where we want the pivot scale to lie $\left(N_{*} \geq N_{m}\right)$.

\subsection{Generating CMB dipole asymmetry}

The relative enhancement in the fast roll scenario contributes an additional factor given by

$$
\frac{\Delta \sigma^{2}}{P_{\delta \sigma_{*}}}=\int_{-\infty}^{N_{m}} 4 e^{2\left(N_{k}-N_{*}\right)+2 \int_{N_{i}}^{0} \Delta \alpha_{\mathbf{k}}(N) \mathrm{d} N} \mathrm{~d} N_{k}
$$

Here we have neglected the integral region $N_{m}<N_{k}<N_{*}$, because this region is not enhanced by the tachyonic fast roll scenario, and has been shown in section 3 to generate only a negligible CMB asymmetry. The inner integral of $\Delta \alpha_{\mathbf{k}}$ is performed for the fast roll phase $N_{i}<N<0$. Then eq. (4.7) can be recast into

$$
\frac{\Delta \sigma^{2}}{P_{\delta \sigma_{*}}}=4 e^{2\left(N_{m}-N_{*}\right)} \int_{-\infty}^{N_{m}} e^{2 \beta\left(N_{k}\right)} \mathrm{d} N_{k}
$$

where we have defined

$$
\beta\left(N_{k}\right) \equiv N_{k}-N_{m}+\int_{N_{i}}^{0} \Delta \alpha_{k}(N) \mathrm{d} N
$$

Since the mode dependence in eq. (4.8) have been absorbed into $\beta\left(N_{k}\right)$, the scale $k_{\max }$ with the largest $\beta\left(N_{k}\right)$ will contribute most to the CMB asymmetry. The overall exponential coefficient in eq. (4.8) simply means that a longer second slow roll phase, which will stretch the initial perturbation modes, leads to a weaker CMB asymmetry. 
We would like $\beta\left(N_{k}\right)$ to peak at some scale $N_{k_{\max }}$, or otherwise it is difficult to produce sufficient CMB dipole asymmetry. The peak mode $N_{k_{\max }}$ can be solved from $\partial \beta\left(N_{k}\right) / \partial N_{k}=$ 0 . Noticing $\Delta \alpha_{\mathbf{k}}(N)$ is only a function of $N-N_{k}$, this yields

$$
\Delta \alpha_{k_{\max }}(0)=\Delta \alpha_{k_{\max }}\left(N_{i}\right)+1 \text {. }
$$

Therefore there would be no peak if $\Delta \alpha_{k}(N)$ is always less than 1 . The above condition requires the tachyonic mass to be heavy enough. According to eq. (4.6), we obtain

$$
m^{2} \geq 2 H^{2} .
$$

The contribution to the CMB asymmetry would then mostly come from around the peak scale $N_{k_{\max }}$. If we know the full width at half maximum (FWHM) of the peak, namely $\Delta N$, we can have a good estimation for the integral, hence writing eq. (4.8) as

$$
\frac{\Delta \sigma^{2}}{P_{\delta \sigma_{*}}} \approx 4 \Delta N e^{2\left(N_{m}-N_{*}+\beta\left(N_{k_{\max }}\right)\right)} .
$$

A typical example of $\beta\left(N_{k}\right)$ is shown in figure 3 , for $N_{i}=-2$ and $N_{m}=1.2$, in which $\beta\left(N_{k}\right)$ peaks at about $N_{k} \approx-1$, with $\beta\left(N_{k_{\max }}\right) \approx 4.7$. The half maximum lies at $\beta\left(N_{k_{\max }}\right)-\frac{1}{2} \ln 2$ with $\Delta N \approx 0.8$. We do not want the pivot scale spectrum to be modified by the fast roll phase, so we yield $N_{*}>N_{m}$, and therefore, we take $N_{*}=N_{m}+1$. Plugging these numbers into eq. (4.12) will give

$$
\frac{|\Delta \sigma|}{\sqrt{P_{\delta \sigma_{*}}}}=72
$$

This result satisfies the necessary condition eq. (2.9), but not eq. (2.7). From this we can see that the bispectrum cancellation can indeed enhance the CMB dipole asymmetry, but this would require another source for the curvature perturbations. However, with a different choice of the parameters $N_{i}=-1.8, N_{m}=1.4$ and $N_{*}=2.4$, it is still possible to satisfy eq. (2.7), as shown in section 6. Also, eq. (4.12) and eq. (4.13) gives the average CMB asymmetry on any direction. When the direction with the strongest asymmetry is chosen, the CMB asymmetry would be larger.

We also have to make sure that the perturbations remain small throughout the dynamics. This typically requires the curvature perturbations generated by the $\sigma$ field to have a power spectrum $P_{\zeta_{\delta \sigma_{k}}}<1$. Since there can be other sources of curvature perturbations, we define a ratio for $\sigma$ at the pivot scale

$$
R^{2} \equiv \frac{P_{\zeta_{\delta \sigma_{*}}}}{P_{\zeta}} \leq 1
$$

The constraint $P_{\zeta_{\delta \sigma *}}<1$ then becomes

$$
\begin{aligned}
P_{\zeta_{\delta \sigma_{k}}} & =\left.P_{\zeta_{\delta \sigma_{k}}}\right|_{s r} e^{2 \int_{N_{i}}^{0} \Delta \alpha_{k}(N) \mathrm{d} N} \\
& =P_{\zeta} R^{2} e^{2 \int_{N_{i}}^{0} \Delta \alpha_{k}(N) \mathrm{d} N}<1,
\end{aligned}
$$

where we have used $\left.P_{\zeta_{\delta \sigma_{k}}}\right|_{s r}=P_{\zeta_{\delta \sigma_{*}}}$ for any mode $k$. This constrains the total amount of enhancement, i.e. the height of the red dashed curve in figure 3 , by

$$
\int_{N_{i}}^{0} \Delta \alpha_{k}(N) \mathrm{d} N<-\frac{1}{2} \ln P_{\zeta}-\ln R, \quad \text { for any } N_{k}<0 .
$$

Because $R \leq 1$ and $P_{\zeta}=2.196 \times 10^{-9}$, in the example, figure 3 , it is easy to see that the red curve is lower than $-\frac{1}{2} \ln P_{\zeta} \approx 10$, and therefore the condition eq. (4.16) is well satisfied. 


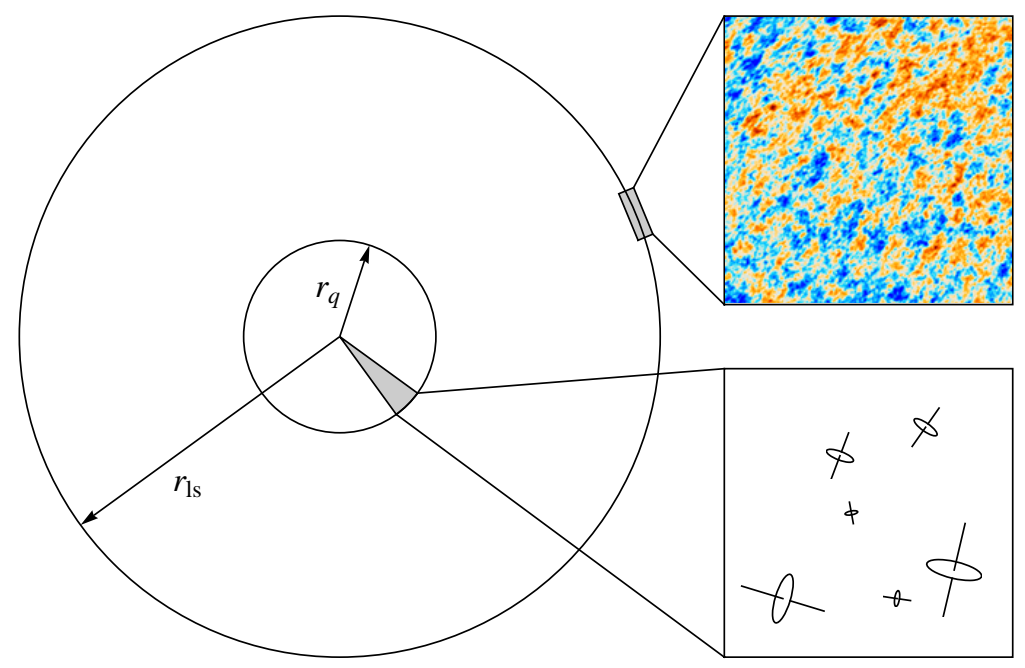

Figure 4. A schematic figure on the dipole asymmetries of the CMB and quasars. The outer sphere is the LSS and the inner one contains all the observed quasars. Therefore the quasar observations can only constrain the asymmetry in the distance scales smaller than $r_{q}$, while the asymmetry in the distance scale $r_{l s}$ can be much larger. In this sense, we need the running in the asymmetry factor $A$ in the length scale in order to satisfy the quasar constraint, which has been observed in the Planck observations in figure 28 of [6], though in $l$ space.

\section{Known constraints}

\subsection{Quasars}

The quasar observations [38] constrain the universe asymmetry in the quasar scale $N_{q}>N_{*}$. If we define $r_{q}$ as our distance to the farthest quasar, we can write $N_{q}$, the length scale of our distance to the quasars, similar with eq. (3.12), as (see also figure 4)

$$
r_{q}=\frac{e^{-N_{q}}}{H}
$$

The quasar observations find no asymmetry, requiring $A<0.02$ in the quasar scale $N_{q}$; see [38]. From eq. (2.6) and eq. (2.8), we find that it can be accommodated with the CMB scale asymmetry $A \sim 0.07$, if the non-Gaussianity parameter, $f_{\mathrm{NL}}$ or $\tau_{\mathrm{NL}}$, has a running. The running should be strong enough to reduce the non-Gaussianity to $\lesssim 1 / 4$ during inflation, from $N=N_{*}$ to $N=N_{q}$.

The amount of non-Gaussianity and its running depend very much on the model, but many existing models can provide such a running. In the spectator model we consider in [28], this can be achieved if the effective mass of the spectator field runs between the Hubble exits of the pivot and quasar scales.

\subsection{Quadrupole and octupole}

The source of CMB asymmetry should not generate excessive quadrupole or octupole in the CMB. Following the conventions in $[7,39]$, we replicate their derived constraints here, from 
eq. (4), and eq. (5) of Ref. [7]

$$
\begin{aligned}
\left(k x_{d}\right)^{2}\left|\Phi_{\vec{k}}\left(\tau_{d}\right) \sin \bar{\omega}\right| & \lesssim 5.8 \mathcal{Q}, & & \text { for quadrupole }, \\
\left(k x_{d}\right)^{3}\left|\Phi_{\vec{k}}\left(\tau_{d}\right) \cos \bar{\omega}\right| & \lesssim 32 \mathcal{O}, & & \text { for octupole }
\end{aligned}
$$

where $\mathcal{Q}=1.8 \times 10^{-5}$ and $\mathcal{O}=2.7 \times 10^{-5}$. We rephrase them with our convention, with $k x_{d}\left|\Phi_{k}\left(\tau_{d}\right)\right|=\frac{1}{3}|\Delta \zeta|=\sqrt{P_{\zeta}} \frac{|\Delta \sigma|}{3 \sqrt{P_{\delta \sigma_{*}}}}$ where $k x_{d}=\sqrt{2} e^{N_{k_{\max }}-N_{*}}$. After neglecting the sin and cos functions, these two inequalities become

$$
\begin{array}{ll}
N_{k_{\max }}-N_{*} \lesssim \ln \frac{17.4 \mathcal{Q}}{\sqrt{2 P_{\zeta}}} \frac{\sqrt{P_{\delta \sigma_{*}}}}{|\Delta \sigma|}, \quad \text { for quadrupole }, \\
N_{k_{\max }}-N_{*} \lesssim \frac{1}{2} \ln \frac{48 \mathcal{O}}{\sqrt{P_{\zeta}}} \frac{\sqrt{P_{\delta \sigma_{*}}}}{|\Delta \sigma|}, & \text { for octupole. }
\end{array}
$$

Therefore the quadrupole and octupole constraints put a lower bound on $N_{*}$, the efolding of the second slow roll phase, see figure 1. In the example shown in figure 3 , we have $N_{k_{\max }} \approx-1$. By plugging in the values of $\mathcal{O}, \mathcal{Q}, P_{\zeta}, N_{*}$ and $|\Delta \sigma| / \sqrt{P_{\delta \sigma_{*}}}$ from the example figure 3 and eq. (4.13), we can see that both the quadrupole and octupole constraints are satisfied.

\section{A viable model}

As we have seen in previous sections that the quasar constraint can always be satisfied with a proper running non-Gaussianity, eq. (4.16) constrains the amount of total enhancement, and the quadrupole and octupole constrain the length of the second slow roll phase. To achieve the maximum CMB dipole asymmetry $A$, we need to maximize the total enhancement and minimize the length of the second slow roll phase. We examine the maximum possible value for $A$ in the following two models mentioned in section 2.

- Inflaton, and the spectator: Inflation is driven by the $\phi$ field, while the curvature perturbations come from $\sigma$, which is a spectator field. The $\sigma$ field generates mild local bispectrum $f_{\mathrm{NL}}=2.7 \pm 11.6$ (at $2 \sigma$ ) [34]. Combining the above constraints gives us an upper bound for the CMB dipole asymmetry

$$
A<1.2 \sqrt{8.7 \mathcal{Q} / \sqrt{2}} \Delta N^{\frac{1}{4}}\left|f_{\mathrm{NL}}\right|,
$$

where we have used the quadrupole constraint, but neglected the octupole because it is much weaker.

After putting in the values for $Q \lesssim 1.8 \times 10^{-5}, \Delta N \approx 1$ and $f_{\mathrm{NL}}<14.3$, we can find the maximum value of $A \lesssim 0.18$, allowed by the constraints. A specific example for this model can be found when $N_{i}=-1.8, N_{m}=1.4, N_{*}=2.4$, and $f_{\mathrm{NL}}=7$, which will generate $A=0.07$. Also, this model can be excluded for $A=0.07$ if the future experiments improve the errors on $f_{\mathrm{NL}}$, so that $\left|f_{\mathrm{NL}}\right| \lesssim 4$. The parameter plot for this model based on eq. (6.1) is shown in figure 5 .

- Inflaton, spectator, and the other: Another possibility is an additional field $\chi$ also contributes to the power spectrum of the curvature perturbations, so $\sigma$ will only 


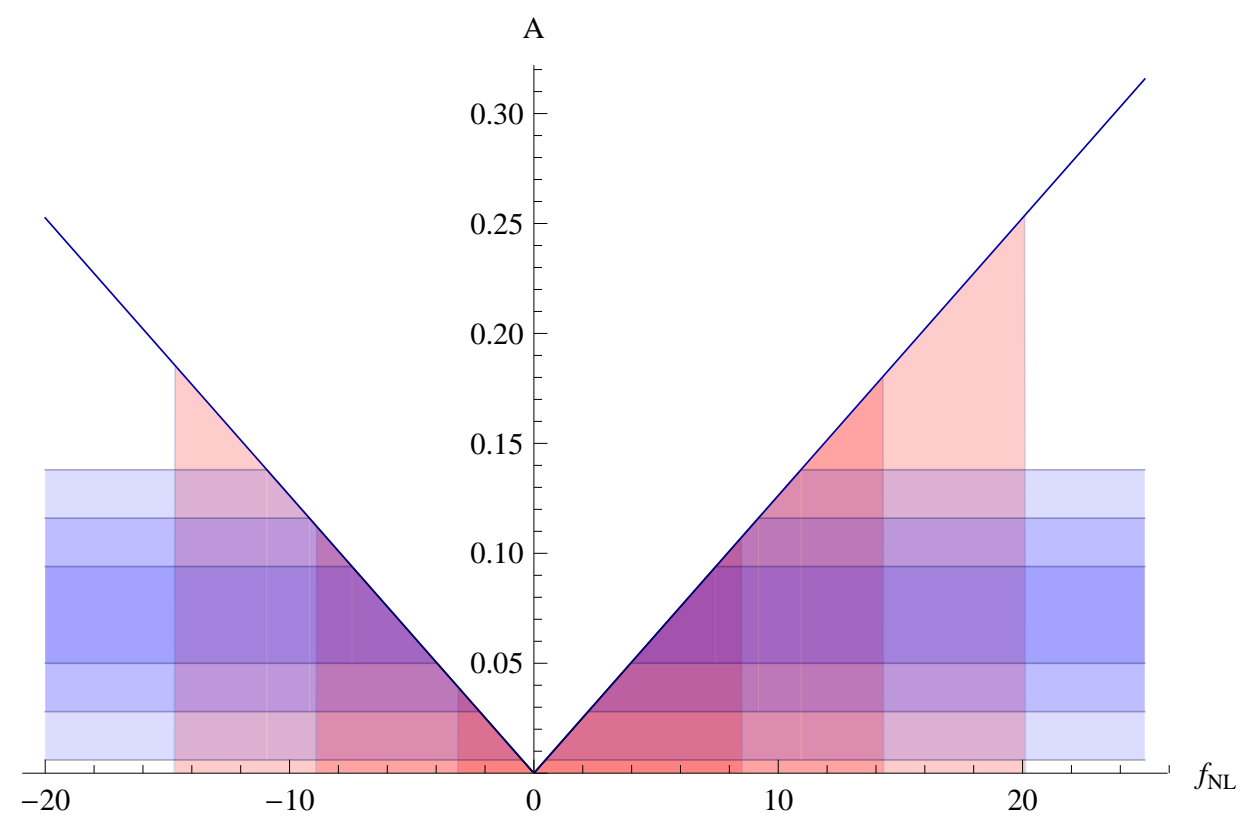

Figure 5. The parameter plot for the inflaton and spectator model in section 6 . The blue line shows the maximum CMB dipole asymmetry can be reached by any bispectrum $f_{\mathrm{NL}}$. The vertical red bands indicate the latest Planck observational bounds for the local bispectrum $f_{\mathrm{NL}}$, for the $1 \sigma, 2 \sigma$ and $3 \sigma$ regions. The horizontal blue bands indicate the Planck observational bounds for the CMB dipole asymmetry, also for the $1 \sigma, 2 \sigma$ and $3 \sigma$ regions. The neighboring area of $f_{\mathrm{NL}} \approx 7$ and $A \approx 0.07$ is within $1 \sigma$ C.L. for both the observables.

contribute the amount $P_{\zeta_{\delta \sigma_{*}}}=R^{2} P_{\zeta}$ where $R<1$. In this case, each of them can generate positive or negative $f_{\mathrm{NL}}$, but in all they cancel. It will produce a stronger dipole asymmetry than the previous case, giving

$$
A<\sqrt{8.7 \mathcal{Q} / \sqrt{2}} \Delta N^{\frac{1}{4}} R^{-\frac{1}{2}} \sqrt{\tau_{\mathrm{NL}}} .
$$

We substitute the values of $Q \lesssim 1.8 \times 10^{-5}, \Delta N \approx 1$ and $\tau_{\mathrm{NL}}<2800$, so the above equation yields $A \lesssim 0.56 R^{-\frac{1}{2}}$. Therefore $A=0.07$ is also allowed, and this model can generate a much stronger dipole asymmetry than the previous one.

\section{Conclusion}

In this paper, we have shown that a spectator field can indeed generate the observed CMB dipole asymmetry. However it requires a brief phase of fast roll before the Hubble exit of the relevant scales to amplify its perturbations. We have shown that this primordial mechanism explains the CMB dipole asymmetry while satisfying all known constraints, the quasar, quadrupole and octupole constraints.

In the inflaton-spectator two-field setup, this mechanism can generate a maximum of $A \lesssim 0.18$, under the current constraint on $f_{\mathrm{NL}}=2.7 \pm 11.6$ (at $2 \sigma$ ). This can be further constrained by future observations, for instance more precise value on $f_{\mathrm{NL}}$. In the three-field setup, the CMB dipole asymmetry $A$ can be enhanced by $\tau_{\mathrm{NL}}$ due to the fine cancellation between the $\pm f_{\mathrm{NL}}$ sources. As a result the asymmetry can be much larger, i.e. $A \lesssim 0.56 R^{-1}$ for $\tau_{\mathrm{NL}}<2800$, where $R$ is defined in Eq. (4.14). 
In the specific examples, we have shown that the CMB dipole asymmetry can still be explained well within an inflation paradigm, but it will require more than one field. We would need a spectator field to generate the curvature perturbations. Single-field inflation is however difficult to generate the observed CMB dipole asymmetry, because of its lack of non-Gaussianity in its simplest form.

\section{Acknowledgments}

We would like to thank Christopher Hirata, Matthew Kleban, David Lyth, John McDonald and Yun-Song Piao for helpful discussions. AM is supported by the

Lancaster-Manchester-Sheffield Consortium for Fundamental Physics under STFC grant ST/J000418/1.

\section{References}

[1] C. Bennett, A. Banday, K. Gorski, G. Hinshaw, P. Jackson, et. al., Four year COBE DMR cosmic microwave background observations: Maps and basic results, Astrophys.J. 464 (1996) L1-L4, [astro-ph/9601067].

[2] G. Hinshaw, D. Larson, E. Komatsu, D. Spergel, C. Bennett, et. al., Nine-Year Wilkinson Microwave Anisotropy Probe (WMAP) Observations: Cosmological Parameter Results, arXiv:1212.5226.

[3] Planck Collaboration Collaboration, P. Ade et. al., Planck 2013 results. XVI. Cosmological parameters, arXiv:1303.5076.

[4] H. Eriksen, F. Hansen, A. Banday, K. Gorski, and P. Lilje, Asymmetries in the Cosmic Microwave Background anisotropy field, Astrophys.J. 605 (2004) 14-20, [astro-ph/0307507].

[5] H. K. Eriksen, A. Banday, K. Gorski, F. Hansen, and P. Lilje, Hemispherical power asymmetry in the three-year Wilkinson Microwave Anisotropy Probe sky maps, Astrophys.J. 660 (2007) L81-L84, [astro-ph/0701089].

[6] Planck Collaboration Collaboration, P. Ade et. al., Planck 2013 results. XXIII. Isotropy and Statistics of the CMB, arXiv:1303.5083.

[7] A. L. Erickcek, M. Kamionkowski, and S. M. Carroll, A Hemispherical Power Asymmetry from Inflation, Phys.Rev. D78 (2008) 123520, [arXiv:0806.0377].

[8] A. L. Erickcek, C. M. Hirata, and M. Kamionkowski, A Scale-Dependent Power Asymmetry from Isocurvature Perturbations, Phys.Rev. D80 (2009) 083507, [arXiv:0907.0705].

[9] Planck Collaboration Collaboration, P. Ade et. al., Planck 2013 results. XXII. Constraints on inflation, arXiv: 1303.5082.

[10] L. Wang, E. Pukartas, and A. Mazumdar, Visible sector inflation and the right thermal history in light of Planck data, arXiv:1303.5351.

[11] A. Mazumdar and J. Rocher, Particle physics models of inflation and curvaton scenarios, Phys.Rept. 497 (2011) 85-215, [arXiv:1001.0993].

[12] R. Allahverdi, K. Enqvist, J. Garcia-Bellido, and A. Mazumdar, Gauge invariant MSSM inflaton, Phys.Rev.Lett. 97 (2006) 191304, [hep-ph/0605035].

[13] R. Allahverdi, K. Enqvist, J. Garcia-Bellido, A. Jokinen, and A. Mazumdar, MSSM flat direction inflation: Slow roll, stability, fine tunning and reheating, JCAP 0706 (2007) 019, [hep-ph/0610134].

[14] R. Allahverdi, A. Kusenko, and A. Mazumdar, A-term inflation and the smallness of neutrino masses, JCAP 0707 (2007) 018, [hep-ph/0608138]. 
[15] S. Choudhury, A. Mazumdar, and S. Pal, Low and High scale MSSM inflation, gravitational waves and constraints from Planck, arXiv:1305.6398.

[16] L. Wang and A. Mazumdar, Small non-Gaussianity and dipole asymmetry in the CMB, arXiv:1304.6399.

[17] L. Dai, D. Jeong, M. Kamionkowski, and J. Chluba, The Pesky Power Asymmetry, arXiv:1303.6949.

[18] D. H. Lyth, The CMB asymmetry from inflation, arXiv:1304.1270.

[19] Z. Chang and S. Wang, Inflation and primordial power spectra at anisotropic spacetime inspired by Planck's constraints on isotropy of CMB, arXiv:1303.6058.

[20] J. McDonald, Isocurvature and Curvaton Perturbations with Red Power Spectrum and Large Hemispherical Asymmetry, arXiv:1305.0525.

[21] M. H. Namjoo, S. Baghram, and H. Firouzjahi, Hemispherical Asymmetry and Local non-Gaussianity: a Consistency Condition, arXiv:1305.0813.

[22] W. Zhao, Directional dependence of CMB parity asymmetry, arXiv:1306.0955.

[23] J. F. Donoghue, K. Dutta, and A. Ross, Non-isotropy in the CMB power spectrum in single field inflation, Phys.Rev. D80 (2009) 023526, [astro-ph/0703455].

[24] X. Chen and Y. Wang, Relic Vector Field and CMB Large Scale Anomalies, arXiv: 1305.4794.

[25] Z.-G. Liu, Z.-K. Guo, and Y.-S. Piao, Obtaining the CMB anomalies with a bounce from the contracting phase to inflation, arXiv:1304.6527.

[26] A. D. Linde, Fast roll inflation, JHEP 0111 (2001) 052, [hep-th/0110195].

[27] A. Mazumdar and L. Wang, Creating perturbations from a decaying field during inflation, Phys.Rev. D87 (2013) 083501, [arXiv:1210.7818].

[28] L. Wang and A. Mazumdar, Cosmological perturbations from a Spectator field during inflation, JCAP 1305 (2013) 012, [arXiv:1302.2637].

[29] T. Moroi and T. Takahashi, Effects of cosmological moduli fields on cosmic microwave background, Phys.Lett. B522 (2001) 215-221, [hep-ph/0110096].

[30] D. H. Lyth and D. Wands, Generating the curvature perturbation without an inflaton, Phys.Lett. B524 (2002) 5-14, [hep-ph/0110002].

[31] K. Enqvist and M. S. Sloth, Adiabatic CMB perturbations in pre - big bang string cosmology, Nucl.Phys. B626 (2002) 395-409, [hep-ph/0109214].

[32] D. H. Lyth, C. Ungarelli, and D. Wands, The Primordial density perturbation in the curvaton scenario, Phys.Rev. D67 (2003) 023503, [astro-ph/0208055].

[33] A. Mazumdar and S. Nadathur, The curvaton scenario in the MSSM and predictions for non-Gaussianity, Phys.Rev.Lett. 108 (2012) 111302, [arXiv:1107.4078].

[34] Planck Collaboration Collaboration, P. Ade et. al., Planck 2013 Results. XXIV. Constraints on primordial non-Gaussianity, arXiv:1303.5084.

[35] K. Enqvist, A. Jokinen, A. Mazumdar, T. Multamaki, and A. Vaihkonen, Non-Gaussianity from preheating, Phys.Rev.Lett. 94 (2005) 161301, [astro-ph/0411394].

[36] K. Enqvist, A. Jokinen, A. Mazumdar, T. Multamaki, and A. Vaihkonen, Non-Gaussianity from instant and tachyonic preheating, JCAP 0503 (2005) 010, [hep-ph/0501076].

[37] A. Jokinen and A. Mazumdar, Very large primordial non-gaussianity from multi-field: application to massless preheating, JCAP 0604 (2006) 003, [astro-ph/0512368].

[38] C. M. Hirata, Constraints on cosmic hemispherical power anomalies from quasars, JCAP 0909 (2009) 011, [arXiv:0907.0703]. 
[39] A. L. Erickcek, S. M. Carroll, and M. Kamionkowski, Superhorizon Perturbations and the Cosmic Microwave Background, Phys.Rev. D78 (2008) 083012, [arXiv:0808.1570]. 